\title{
Michel Borghini as a Mentor and Father of the Theory of Polarization in Polarized Targets
}

\author{
Wim de Boer \\ Karlsruhe Institute of Technology, Physics Department, IEKP, Campus Süd, \\ Postfach 6980, 76049 Karlsruhe, Germany \\ wim.de.boer@kit.edu
}

Published 29 February 2016

\begin{abstract}
This paper is a contribution to the memorial session for Michel Borghini at the Spin 2014 conference in Bejing, honoring his pivotal role for the development of polarized targets in high energy physics. Borghini proposed for the first time the correct mechanism for dynamic polarization in polarized targets using organic materials doped with free radicals. In these amorphous materials the spin levels are broadened by spin-spin interactions and g-factor anisotropy, which allows a high dynamic polarization of nuclei by cooling of the spin-spin interaction reservoir. In this contribution I summarize the experimental evidence for this mechanism. These pertinent experiments were done at CERN in the years 1971 - 1974, when I was a graduate student under the guidance of Michel Borghini. I finish by shortly describing how Borghini's spin temperature theory is now applied in cancer therapy.
\end{abstract}

Keywords: Dynamc polarization; spin temperature theory; cancer therapy.

\section{Introduction}

Studying the effect of spin in particle interactions has been a topic of interest in high energy physics (see e.g. Ref. ${ }^{1}$ for a review), which required polarized particles, either as target or as beam or both. The development of polarized targets at CERN was driven by Michel Borghini, while Alan Krisch from the University of Michigan (Ann Arbor) pushed the polarized beams at Argonne and later at Brookhaven and other accelerators. $^{2}$ I was lucky enough to work with both of them. After finishing my Master thesis at the Technical University of Delft on studying spin systems in $\mathrm{LMN}^{3}$ a material used initially for polarized targets, I came to CERN as a fellow in Michel Borghini's group and contributed heavily to the experiments leading to the acceptance of Borghini's mechanism of dynamic nuclear polarization (DNP) in organic materials ${ }^{4,5}$ by "Dynamic Orientation of Nuclei by Cooling of the Electron

This is an Open Access article published by World Scientific Publishing Company. It is distributed under the terms of the Creative Commons Attribution 3.0 (CC-BY) License. Further distribution of this work is permitted, provided the original work is properly cited. 
Spin-Spin Interactions" a . Here the spin-spin interactions (SS) comprise all the nonZeeman energies, which can broaden the Zeeman levels of the free electrons beyond the nuclear Zeeman levels, thus allowing a thermal contact between the SS-reservoir and the nuclear Zeeman reservoir by electron spin flips in combination with nuclear spin flips. Such a thermal contact is driven at low temperatures mainly by induced spin flips from the polarizing RF field. This dual role of the external RF field (cooling and establishing thermal contact) at low temperatures and in high magnetic fields was the main new idea from Michel, since DNP by cooling of the spin-spin interactions had been demonstrated before in 1963 by Goldman and Landesman ${ }^{6}$ in the group of A. Abragam, the world leading expert on DNP at Saclay. but its application to amorphous materials at low temperatures was far from clear.

Borghini, also working in Abragam's group, wrote down his ideas in an extensive thesis. However, his thesis was not accepted by Abragam for reasons unkown to me, but presumably because it lacked experimental verification. Michel's proposed mechanism was clearly proven by our experiments at CERN, done at temperatures down to $0.1 \mathrm{~K}$ and magnetic fields up to $5 \mathrm{~T}$. After all our results were published by 1976, ${ }^{7-13}$ Abragam and Goldman wrote a review on DNP, describing in detail our results and recognizing that this was the mechanism of DNP in polarized targets. ${ }^{14}$ These papers were the basis of my PhD thesis ${ }^{15}$ at the Technical University of Delft. Promotor was Prof. B.S. Blaisse and Michel was a member of the thesis committee, as shown in Fig. 1.

When I came to Borghini's group in 1971, scattering experiments with polarized butanol targets ${ }^{16}$ were in full swing. However, a higher proton polarization was requested and we continued to work on Michel's list of possible materials, which should be tried. This was extremely tedious, since every material could be doped with every free radical in a range of concentrations. Michel left every one much freedom in trying out ideas and organizing his work. This fosters the creativity of the individual much stronger than in a hierarchical group structure, where everyone is told what to do. I have kept this working style in my working groups. We rarely had group meetings, but Michel regularly informed himself how things were going and took care that the infrastructure was optimal, so we had an outstanding mechanical workshop with Jean-Michel Rieubland as driving force behind the actual building and running of the polarized targets, George Gattone as head of the chemistry laboratory, Fred Udo and Huib Ponssen, also two dutch staff members, providing the electronics and digital readout of the polarization. It included for every target an HP2100 computer, which allowed not only a precision determination of the polarization by averaging the sometimes tiny thermal equilibrium signals, but I could also do all calculations for my thesis on my personal computer. The programs were punched with a Teletype writer on paper rolls, which in turn could be read by optical readers. All this was high tech at that time. Then there was of

a Michel called this the "DONKEY" effect, but the name did not stick. 

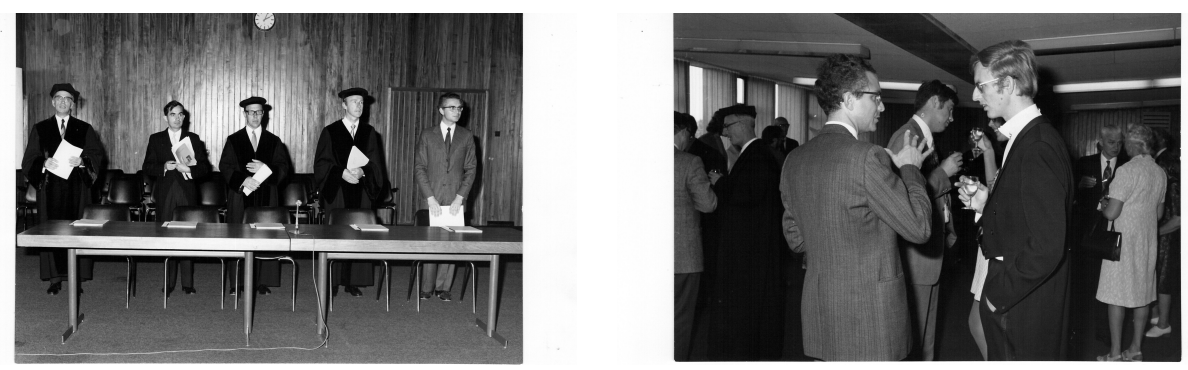

Fig. 1. a) My thesis Committee at the Technical University of Delft (1974) with Michel on the right. b) Discussion with Michel after the thesis exam.

course Tapio Niinikoski, the cryogenic genius, who obtained his thesis on the development of the horizontal dilution refrigerators in the group of Prof. O.V. Lounasma at the Helsinki University of Technology, roughly at the same time as I received my Ph.D thesis at the Technical University of Delft. Tapio was the mastermind behind the frozen spin targets ${ }^{17}$ and became the head of the polarized target group after Michel took over other responsibilities at CERN. After getting to know all the tricky details of how to build and operate a polarized target, I was hired by Alan Krisch at the University of Michigan in Ann Arbor, who had just started to do experiments with polarized beams at Argonne National Laboratory and installed a polarized target. This enabled us to measure the cross section for a polarized proton beam on a polarized proton target. Surprisingly, this led to significant spin effects, even in the total elastic pp cross section for protons with spins parallel or antiparallel, an experimental result, ${ }^{18}$ which still lacks an interpretation in the frame work of QCD. But for me the main result was, that high energy physics is at least as interesting as solid state physics, so I became a particle physicist ${ }^{\mathrm{b}}$. In this contribution I want to summarize the exciting experiments done between 1971 and 1974 in Borghini's group at CERN.

\section{The Theory of Dynamic Polarization}

In solid crystalline materials doped with a small concentration of paramagnetic centers with an unpaired free electron the mechanism of dynamic polarization is easy to understand: in a magnetic field $\mathrm{H}$ at a temperature $\mathrm{T}$ the relative fraction of the free electrons $n_{i}$ over the spin states with energy $E_{i}$ is given by the Boltzmann distribution $n_{i}=\exp \left(-E_{i} / k T_{S}\right)$ for a spin temperature $T_{S}$. This leads for a spin $1 / 2$ to a polarization $P=\left(n_{+}-n_{-}\right) /\left(n_{-}+n_{-}\right)=\tanh \left(h \nu / 2 k T_{S}\right)$, where $h$ is Planck's constant and $\nu$ the Larmor frequency of the spin system. At high temperatures one can expand the exponential expressions, in which case the electron and nuclear

${ }^{\mathrm{b}}$ The possible unification of all forces in Supersymmetry ${ }^{19}$ spurred my interest in dark matter, ${ }^{20}$ so I later joined, in addition, the astroparticle physics community in search for the elusive dark matter. 


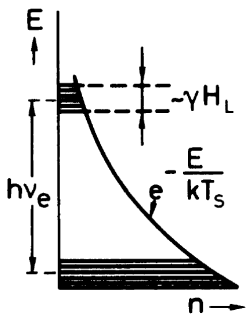

a)

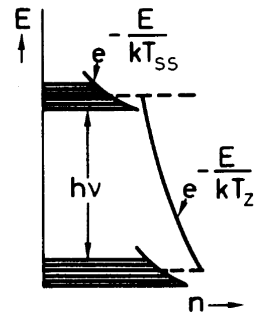

b)

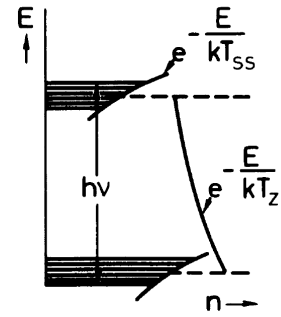

c)

Fig. 2. a) The equilibrium spin temperature $T_{S}$ determines the population of the energy levels in a magnetic field. Off-resonance microwave radiation below (above) the Larmor frequency populates the lower (upper) levels inside the broadened Zeeman levels, thus cooling (heating) the SS-reservoir, as indicated in b) (c). This leads to different temperatures of the Zeeman - and SS-reservoir, indicated by $T_{Z}$ and $T_{S} S$, respectively.

polarization are related to the inverse spin temperature $\beta=h / k T_{S}$ by $P_{e}=-\beta \nu_{e} / 2$ and $P_{n}=\beta \nu_{n} / 2$. Numerical results for the Larmor frequency and a spin temperature $T_{S}$ equal to the lattice temperature lead to an electron polarization $P_{e}=-0.9975 \mathrm{in}$ a magnetic field of $2.5 \mathrm{~T}$ and a temperature $\mathrm{T}$ of $0.5 \mathrm{~K}$, while the proton polarization $P_{n}=+0.00511$ under the same conditions. DNP is the art of transferring the high electron polarization to the nuclei via microwave induced spin flips.

For crystalline materials the dominant DNP mechanism is the "solid" effect (also called solid-state effect), which was proposed by Abragam and Proctor and verified experimentally, see the review ${ }^{14}$ for original references. In this case one stimulates by microwave irradiation the "forbidden" transitions, in which case an electron and neighboring nucleus simultaneously change their spin orientation (either flip-flip or flip-flop transitions, where a flip (flop) indicates a transition to a higher (lower) Zeeman level). The electron will return to the ground state quickly with a time constant given by the short electron spin-lattice relaxation time of the order of ms. The nucleus has a much longer spin lattice relaxation time, so it does not quickly return to the ground state, but instead it can transfer its polarization to neighboring nuclei via flip-flop spin transitions. This leads to spin diffusion, which is fast, since energy and angular momentum are conserved. The electron is now ready to polarize the neighboring nucleus again, if it is still receiving photons with the correct energy from an external microwave field. This combination of an external microwave field polarizing neighboring nuclei combined with fast nuclear spin diffusion allows to effectively transfer the high polarization from the electrons to the nuclei.

In non-crystalline solids the spin levels are usually broadened by the different orientations of the molecules, which experience different internal magnetic fields and this broading is usually larger than the Zeeman splitting of the nuclei. In this case the resolved solid-state effect will not work anymore, since one is stimulating 


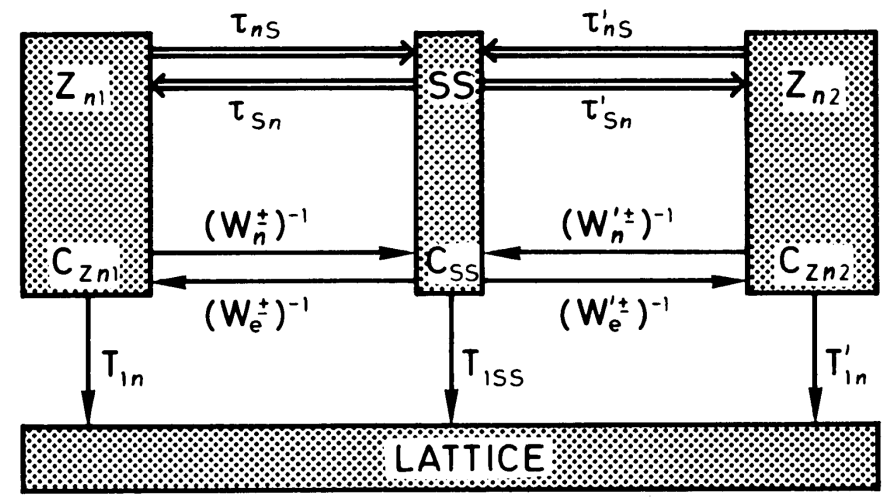

Fig. 3. Schematic diagram of the thermal contact between two nuclear Zeeman reservoirs $Z_{n i}$ with heat capacities $C_{Z n i}$, the spin-spin interaction reservoir $S S$ and the lattice. The double arrows indicate the thermal contact via the flip-flop transitions between two electrons accompanied with a nuclear spin flip, while the single arrows indicate the microwave induced forbidden transitions of a simultaneous electron and nuclear spin transition.

simultaneously flip-flop and flip-flip transitions ${ }^{\mathrm{c}}$. However, another mechanism of DNP may become effective, which is most easily explained by first introducing the concept of a spin temperature and a spin-spin interaction (SS) reservoir. ${ }^{6,21}$ These concepts are visualized in Fig. 2. In a) the populations of the energy levels follow the Boltzmann distribution, both, for the large Zeeman splitting of the electrons and inside an energy band for a given Zeeman level. However, inside a band, whose width is determined by the non-Zeeman interactions, like the g-factor anisotropy or spin-spin interactions (SS), the population can be changed by external photons, if one irradiates with microwave frequencies slightly different from the central Zeeman frequency. This can either cool (Fig. 2b) or heat (Fig. 2c) the SS-reservoir ${ }^{22,23}$ and lead even to the highest levels having the highest population, as shown in Fig. 2c, which corresponds to negative spin temperatures of the SS-reservoir. The question is: how strong is the thermal contact between the nuclear Zeeman energy reservoir and this SS-reservoir? This contact can be established either by (i) spontaneous electron spin flip-flops between the Zeeman levels with a simultaneous nuclear spin flip (so a 3-spin process) or this contact can be established by (ii) the microwave induced forbidden transitions of the "solid" effect. The different transitions for the thermal contact are schematically indicated in Fig. 3. The thermal contact via (i) was demonstrated by Goldman and Landesman, ${ }^{6}$ who first cooled the SS-reservoir by off-resonance RF irradiation. They then switched on a magnetic field, which revealed a nuclear polarization, obviously obtained from the thermal contact with

\footnotetext{
${ }^{\mathrm{c}}$ The net polarization is then given by the difference in intensity of the flip-flop and flip-flip transitions, which is proportional to the difference in intensity of the electron spin resonance line shape and is called the differential solid effect. It always leads to a nuclear polarization well below the electron polarization.
} 


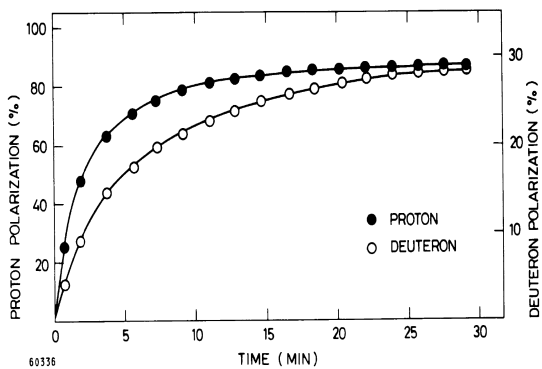

a)

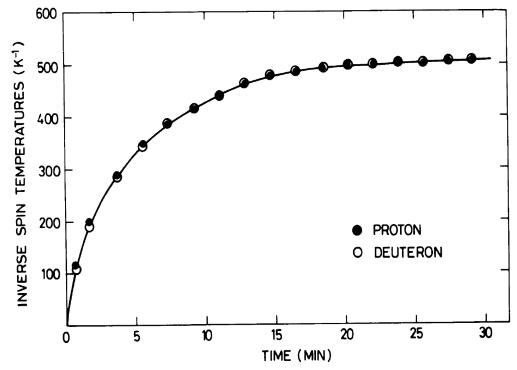

b)

Fig. 4. a) Build-up of the proton and deuteron polarization (indicated on the left- and right-hand scale, resp.) in a partially deuterated sample. b) Build-up of the spin temperature of protons and deuterons.

the SS-reservoir. However, this method is unlikely to function well at low temperatures, since then all electron spins are in the lowest state, so there will be few double spin flips between the Zeeman levels of the electrons. Here came the excellent idea of Borghini: ${ }^{4,5}$ he realized that the second method of a thermal contact is independent of the temperature, so it will be the dominant method at low temperature. So he extended the Provotorov rate equations ${ }^{24}$ to include the nuclei and solved the three coupled differential equations for the temperatures of the SS- and Zeeman reservoirs of electrons and nuclei. The master equation was well explained by Borghini in his rejected thesis and I repeated the proof in the appendix of my thesis. ${ }^{15}$ However, the formulae were written in the high temperature approximation, i.e. expanding the exponential function in the Boltzmann distribution. I extended the differential rate equations from Provotorov to low temperatures. The solutions could still be written analytically, but they were most easily solved numerically. Given that we obtained spin temperatures as low as a few $\mu K$, the precision had to be better than $10^{-10}$, which I could nicely do on "my" HP2100.

\section{Verifying the Mechanism of DNP in Polarized Targets}

The first polarized targets consisted of frozen butanol beads doped with a free radical and reached a proton polarizations of about $40 \% .{ }^{16} \mathrm{~A}$ few years later propanediol doped with $\mathrm{Cr}-\mathrm{V}$ complexes were used, in which a proton polarization close to $100 \%$ was obtained. ${ }^{7,8}$ Such a high polarization would be impossible for the differential solid-state effect. So only the cooling via the SS-reservoir remained a possibility and we started a program to prove this. The predictions of Borghini's spin temperature model are clear: several nuclear species with different Larmor frequencies obtain a different polarization, but they have the same spin temperature, if the thermal contact is good enough, where good enough means that the leakage to the lattice is small in comparison with the heat transfer between the reservoirs in Fig. 3. This 


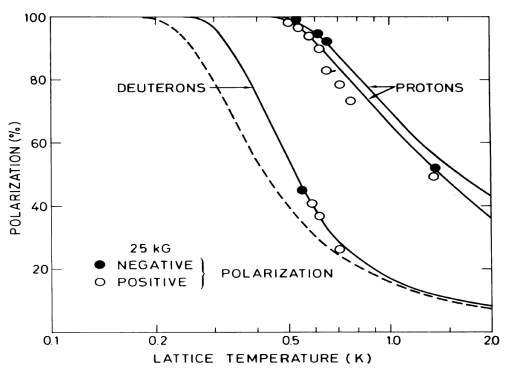

a)

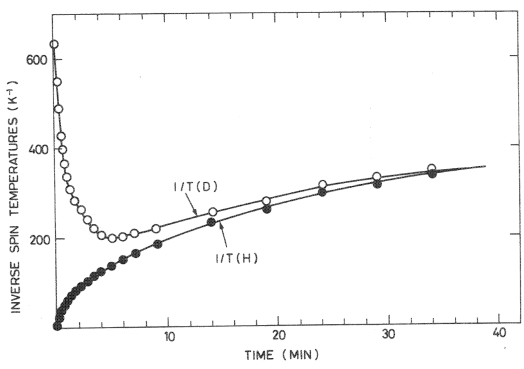

b)

Fig. 5. a) The observed and calculated polarization of protons and deuterons as function of the lattice temperature. b) Evolution of the spin temperature of protons and deuterons after destroying the proton polarization and switching on a microwave field to polarize again. The rapid equalization of the spin temperatures shows the increase of the thermal contact via the forbidden transitions (single arrows in Fig. 3). They finally reach the common temperature $T_{S S}$ of the spinspin interaction reservoir.

could be verified by observing the proton and deuteron polarization in a partially deuterated propanediol sample, as demonstrated in Fig. 4. During the polarization build-up the polarization becomes different (left side), but the spin temperature of the two nuclear species stays the same (right side). In the thermodynamical model of Fig 3 the final polarization depends on the leakage to the lattice, which is a strong function of temperature. ${ }^{8}$ Fig. 5a shows the predicted and observed polarization of protons and deuterons in propanediol doped with $\mathrm{Cr}-\mathrm{V}$ complexes as function of temperature. ${ }^{13}$ Satisfactory agreement between theory and experiment is obtained. Here the heat capacities of the nuclear Zeeman reservoirs and temperature dependence of the spin-lattice relaxation times were carefully taken into account. The effect of the reduced heat capacity of the deuteron system is clearly seen by the difference between the dashed and solid line for the deuterons. At low temperatures the nuclear Zeeman reservoirs in Fig. 3 are rather isolated, at least without microwave irradiation establishing a thermal contact (single arrows in Fig. 3). This allows to destroy the polarization of one nuclear species by inducing spin transitions between the lower and upper nuclear Zeeman levels with a saturating RF field. If one e.g. destroys the proton polarization in a highly polarized sample, the deuteron polarization stays high. After switching on the microwave irradiation to polarize the sample again, this microwave irradiation establishes the thermal contact between the protons and deuterons, thus equalizing their spin temperatures much faster than expected from the polarization time by cooling of the SS reservoir. This is demonstrated in Fig. 5b, which clearly proves the dual role of the microwave field at low temperatures, the original idea of Michel.

Since the deuteron Larmor frequency is smaller than the width of the proton Zeeman levels, one has the same situation between deuterons and protons as for protons and electrons. Therefore, one should be able to polarize the deuterons by 


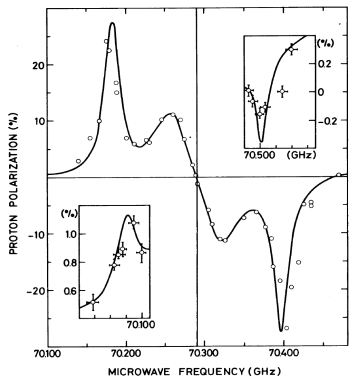

a)

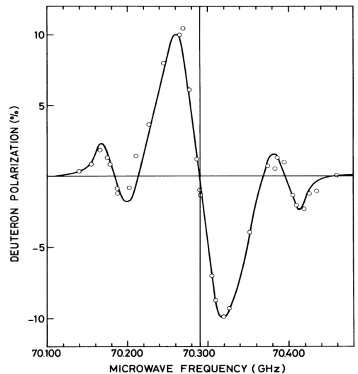

b)

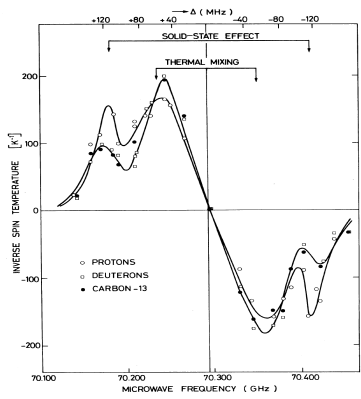

c)

Fig. 6. a) Proton polarization as function of microwave frequency. b) Deuteron polarization as function of microwave frequency. c) Inverse spin temperature as function of microwave frequency.

off-center irradiation of a polarized proton spin system, thus cooling the SS-reservoir of the protons. Several schemes are possible: first polarize a sample of protons and deuterons by cooling the SS-reservoir of the electrons via off-center irradiation of the electron Zeeman levels with microwaves. Then destroy the deuteron polarization with a saturating RF field, followed by a cooling of the proton SS-reservoir with another RF field close to the proton Larmor frequency. Since the deuteron has $\mathrm{S}=1$ the various levels can be populated such, that a pure tensor polarization can be obtained. Many experiments have been done and they all confirm the spin temperature theory in a quantitative way. ${ }^{9,11}$ Because of lack of space these beautiful experiments will not be described here.

\section{Experiments Showing Different Mechanisms of DNP}

As mentioned before, the solid-state mechanism of DNP is effective for narrow Zeeman energy levels of the unpaired electrons of the free radicals, while the mechanism via cooling of the spin-spin interaction reservoir is effective for electron Zeeman levels broader than the nuclear Zeeman splitting. The free radical BDPA has a width of the Zeeman levels, which is below the nuclear Zeeman levels of protons, but above the ones for deuterons and ${ }^{13} \mathrm{C}$ nuclei. Therefore one expects a combination of the two mechanisms, which should occur at different microwave frequencies. This was indeed the case as proven in a frozen sample of partially deuterated m-xylene $(2,2$ D6) doped with BDPA $\left(6 \cdot 10^{18}\right.$ spins $\left./ \mathrm{cm}^{3}\right)$. The measurements were performed in a magnetic field of $2.5 \mathrm{~T}$ at a temperature of $0.75 \mathrm{~K} .{ }^{12,13,15}$ The proton polarization is shown as function of microwave frequency in Fig. 6a. The inner peaks correspond to the polarization by the cooling of the SS-reservoir, while the outer peaks at frequencies $\nu_{e} \pm \nu_{p}$ correspond to the solid-state effect. The insets show the double solid-state effect at frequencies $\nu_{e} \pm 2 \nu_{p}$ corresponding to a simultaneous spin flip of an electron and two protons. Deuterons have a Larmor frequency below the width of the electron Zeeman levels, so the polarization by the forbidden transitions of an 
electron and deuteron spin flip are not visible, since they are too close to the frequencies of the optimum cooling of the electron SS-reservoir. However, the double solid state effect of a simultaneous triple spin flip of an electron, proton and deuteron at frequencies $\nu_{e} \pm \nu_{p} \pm \nu_{D}$ leads to the four peaks in the deuteron polarization outside the main peak from the cooling of the SS-reservoir in Fig. 6b. The maximum polarization of protons and deuterons by cooling of the SS-reservoir (inner peaks in Figs. $6 \mathrm{a}$ and b) is about 10\%. From a comparison with Fig. 4 it is clear that this does not correspond to an equal spin temperature, presumably because of the poor thermal contact between the proton Zeeman reservoir and the SS-reservoir. A good thermal contact requires the width of the electron Zeeman levels to be large with the nuclear Zeeman splitting, which is the case for the deuteron system, but not for the proton spin system. To check this hypothesis we prepared a sample of toluol-D8 with a larger concentration of BPA $\left(5 \cdot 10^{19}\right.$ spins $\left./ \mathrm{cm}^{3}\right)$, which increases the width of the electron Zeeman levels. In addition, we measured the polarization of the ${ }^{13} \mathrm{C}$ nuclei, which have a small Zeeman splitting as well and hence, should obtain the same spin temperature as the deuterons. This is indeed the case, as shown in Fig. 6c. The protons obtain indeed almost the same spin temperature with this sample with an order of magnitude higher concentration of BPDA leading to a broadening of the electron Zeeman levels.

\section{Application of DNP in Cancer Therapy}

Dynamic polarization has found an actively pursued application in cancer research: polarized ${ }^{13} \mathrm{C}$ nuclei in tracers of tumors yield a strongly enhanced signal in Magnetic Resonance Imaging (MRI), so smaller tumors can be discovered, see Ref. ${ }^{25}$ for a recent development and references therein. The medical people call this hyperpolarization, but the polarization happens in setups similar to the ones used in polarized targets, see e.g.. ${ }^{26}$ The surprising discovery: after thawing the samples in a magnetic field, the polarization is largely maintained in the gas phase. Dissolving the gas into a liquid and injecting it into the body yields strongly enhanced NMR signals of the tumors. The polarization lasts only minutes, but this is enough for a picture in a modern magnetic resonance tomograph.

\section{Summary}

The mechanisms of dynamic polarization in polarized targets are by now well understood. According to Michel Borghini's idea this happens via a two-step process between the different heat reservoirs: i) cooling or heating of the SS-reservoir by off-center microwave irradiation; ii) establishing thermal contact between the SS-reservoir and the nuclear Zeeman reservoirs by the same microwave irradiation inducing triple spin-flips, namely a flip-flop transition of two electrons combined with a nuclear spin transition (either up or down until thermal equilibrium is reached). This mechanism was proven by many different experiments showing the thermal contact between the different reservoirs. By extending the usual high 
temperature approximation to low temperatures the spin temperature theory was proven to be valid to spin temperatures in the $\mu \mathrm{K}$ range, as was evident from the excellent agreement between theory and experiment. Nowadays the polarized targets, invented for high energy experiments, are used to enhance the polarization in biological tracers used to find tumors in Magnetic Resonance Imaging. The enhanced polarization provides a strongly enhanced signal, thus allowing to detect smaller tumors. The relatively high ${ }^{13} \mathrm{C}$ polarization of up to $60 \%$ is a clear manifestation, that Borghini's proposed mechanism of the dynamic polarization by cooling of the electron spin-spin interaction reservoir is at work. Michel certainly would have been delighted to see that his idea of dynamic polarization has found such important applications in fields never thought of before.

\section{References}

1. R. G. Milner, PoS PSTP2013, p. 3 (2013).

2. A. Krisch, 13th Workshop on High Energy Spin Physics (DSPIN-09), Dubna, Russia, C09-09-01 (2010).

3. R. de Beer, W. de Boer, C. van 't Hof and D. van Ormondt, Acta Crystallogr.B Struct.Crystallogr.Cryst.Chem. 29, 1473 (1973).

4. M. Borghini, Phys.Lett. 26A, p. 242 (1968).

5. M. Borghini, Proc. 2nd International Conference on Polarized Targets, Berkeley, CA, USA (Ed. G. Shapiro) C710830, 1 (1971).

6. M. Goldman and A. Landesman, Phys.Rev. D132, p. 610 (1963).

7. W. de Boer, Nucl.Instrum.Meth. 107, 99 (1973).

8. W. de Boer and T. Niinikoski, Nucl.Instrum.Meth. 114, 495 (1974).

9. W. de Boer, M. Borghini, K. Morimoto, T. Niinikoski and F. Udo, Phys.Lett. B46, 143 (1973).

10. M. Borghini, W. de Boer and K. Morimoto, Phys.Lett. A48, p. 244 (1974).

11. W. de Boer, Phys.Rev. B12, 828 (1975).

12. M. Borghini, W. de Boer and K. Morimoto, Phys.Lett. A48, p. 244 (1974).

13. W. de Boer, J.Low. Temp.Phys. 22, p. 185 (1976).

14. A. Abragam and M. Goldman, Rep. Prog. Phys. 41, p. 395 (1978).

15. W. de Boer, PhD thesis, Tech. Univ. of Delft, CERN Yellow Report, CERN-7411 (1974), http://cds.cern.ch/record/186203/files/CERN-74-11.pdf.

16. S. Mango, O. Runolfsson and M. Borghini, Nucl.Instrum.Meth. 72, 45 (1969).

17. T. Niinikoski and F. Udo, Nucl.Instrum.Meth. 134, p. 219 (1976).

18. W. de Boer, R. C. Fernow, A. Krisch, H. Miettinen, T. Mulera et al., Phys.Rev.Lett. 34, 558 (1975).

19. U. Amaldi, W. de Boer and H. F'urstenau, Phys.Lett. B260, 447 (1991).

20. W. de Boer, Prog.Part.Nucl.Phys. 33, 201 (1994).

21. I. Solomon, Proc. Magnetic and Electric Resonance and Relaxation, Amsterdam, Ed. J. Schmidt, p. 25 (1963).

22. A. Redfield, Phys.Rev. 98, p. 1787 (1955).

23. B. N. Provotorov, JETP 14, p. 1126 (1961).

24. B. N. Provotorov, JETP 15, p. 611 (1962).

25. T. Rodrigues, E. Serrao, B. W. C. Kennedy, D. Hu, K. M. I. and K. Brindle, Nature Medicine 20, p. 9397 (2014).

26. T. Eichhorn, Y. Takado, N. Salameh et al., Proc. of the Nat. Acad. of Science of the USA 110(45), 18064 (2013). 